\title{
Preparation of Biological Samples Containing Metoprolol and Bisoprolol for Applying Methods for Quantitative Analysis
}

\author{
Mahu Ștefania Corina ${ }^{1 *}$, Hăncianu Monica², Agoroaei Luminița ${ }^{1}$, Coman Băbușanu Anda Cristina1, \\ Butnaru Elena1
}

1 "Grigore T. Popa" University of Medicine and Pharmacy lași, Faculty of Pharmacy, Department of Toxicology, lași, România

2 "Grigore T. Popa" University of Medicine and Pharmacy- lași, Faculty of Pharmacy, Department of Pharmacognosy, lași, România

\begin{abstract}
Arterial hypertension is a complex disease with many serious complications, representing a leading cause of mortality. Selective beta-blockers such as metoprolol and bisoprolol are frequently used in the management of hypertension. Numerous analytical methods have been developed for the determination of these substances in biological fluids, such as liquid chromatography coupled with mass spectrometry, gas chromatography coupled with mass spectrometry, high performance liquid chromatography. Due to the complex composition of biological fluids a biological sample pre-treatment before the use of the method for quantitative determination is required in order to remove proteins and potential interferences. The most commonly used methods for processing biological samples containing metoprolol and bisoprolol were identified through a thorough literature search using PubMed, ScienceDirect, and Willey Journals databases. Articles published between years 2005-2015 were reviewed. Protein precipitation, liquid-liquid extraction and solid phase extraction are the main techniques for the extraction of these drugs from plasma, serum, whole blood and urine samples. In addition, numerous other techniques have been developed for the preparation of biological samples, such as dispersive liquid-liquid microextraction, carrier-mediated liquid phase microextraction, hollow fiber-protected liquid phase microextraction, on-line molecularly imprinted solid phase extraction. The analysis of metoprolol and bisoprolol in human plasma, urine and other biological fluids provides important information in clinical and toxicological trials, thus requiring the application of appropriate extraction techniques for the detection of these antihypertensive substances at nanogram and picogram levels.
\end{abstract}

Keywords: selective beta-blockers, protein precipitation technique, liquid-liquid extraction, solid phase extraction.

Received: 30 April 2015 / Accepted: 18 July 2015

\section{Introduction}

Hypertension is one of the most common diseases in the world, being a constant concern for researchers due to cardiovascular complications and high mortality rate [1].

Selective beta-blockers are clinically important, being frequently used in the treatment of cardiovascular diseases [2]. They were initially used in the management of cardiac arrhythmias, for cardioprotection after myocardial infarction, and subsequently administered in case of hypertension [3].

Metoprolol and bisoprolol are both used in the treatment of arterial hypertension (Figure 1). Metoprolol, (RS)1-(isopropylamino)-3-[4-(2-methoxyethyl)-phenoxy]-propan-2-ol, it is a lipophilic compound with short half-life. The S-enantiomer is responsible for the beta-blocking activity. Also, it is the predominant form in humans, with a plasma ratio of $S / R$-metoprolol $>1$. The selective monitoring of the enatiomers is important for clinical studies of metoprolol. Metoprolol is metabolized by cytochrome P450 (CYP) 2 D6 and shows two active metabolites, O-desmethylmetoprolol and $\alpha$-hydroxymetoprolol. In the past 25 years metoprolol has been used as an ideal probe drug for

* Correspondence to: Stefania-Corina Mahu

E-mail: stefania.mahu@yahoo.com phenotyping study because of its high safety and wide availability [2]. Bisoprolol, (RS)-1-\{4-[(2-isopropoxyethoxy)methyl]-phenoxy\}-3-(isopropylamino)-propan-2-ol, is also frequently administered to patients with hypertension. It<smiles>COCCc1ccc(OCC(O)CNC(C)C)cc1</smiles>

Metoprolol<smiles>CC(C)NCC(O)COc1ccc(COCCOC(C)C)cc1</smiles>

Bisoprolol

Fig.1. Structures of metoprolol and bisoprolol. 
has decreased incidence of central nervous system side effects because is less lipophilic than metoprolol. It works by lowering blood pressure and myocardial contractile force. At the same time, it slows the heart rate being also used in the treatment of cardiac rhythm disorders [4].

The determination of these drugs in biological fluids is essential in toxicological and clinical studies [5], therapeutic monitoring, anti-doping control and in forensics [6].

Numerous analytical methods had been developed for the determination of metoprolol and bisoprolol in biological fluids such as plasma, whole blood, urine. These methods include liquid chromatography coupled with mass spectrometry (LC-MS/MS) [7-17], liquid chromatography-electrospray ionization-mass spectrometry (LC-ESI-MS/MS) [18,19], gas chromatography coupled with mass spectrometry (GC-MS) [3,20-22], high performance liquid chromatography (HPLC) [23,24], high performance liquid chromatography coupled with mass spectrometry (HPLC-MS) [25,26], high performance liquid chromatography with fluorescence detection (HPLCFLD) $[2,27,28]$, high performance liquid chromatography coupled with ultraviolet detection (HPLC-UV) [29], reversed-phase ultra-high-performance liquid chromatography coupled with mass spectrometry (UHPLC-MS) [30].

A limited number of analytical methods are sensitive enough for directly identifying drug molecules in biological samples [31]. As biological fluids have a complex composition, a biological sample pre-treatment before applying the method for quantitative evaluation is required to remove proteins and potential interferences [11]. The main techniques for processing biological sample containing selective beta-blockers, such as metoprolol and bisoprolol, were identified through a thorough literature search of PubMed, ScienceDirect, and Willey Journals databases. Articles published between years 2005-2015 were reviewed.

\section{Biological sample preparation}

Protein precipitation, liquid-liquid extraction and solid phase extraction are the main extraction techniques used for the separation of metoprolol and bisoprolol in plasma, serum, whole blood and urine samples. The small amount of analytes at nanogram and picogram levels led to the development of new techniques for biological sample processing, such as dispersive liquid-liquid microextraction (DLLME) [29], carrier-mediated liquid phase microextraction (CM-LPME) [24], hollow fibre-protected liquid phase microextraction (HF-LPME) [21] and on-line molecularly imprinted solid phase extraction [32].

In the separation of metoprolol and bisoprolol from biological samples an important role plays the extraction solvent which has a high influence on the percentage of recovery. It is important to know its density, its solubility in water and also the extraction capacity of the selected betablockers. The extraction process can be also influenced by the extraction solvent volume. A large volume of extraction solvent decrease the sensitivity due to the dilution effect. Because both the analytes are soluble in water it is important to use a salting-out agent to reduce the water solubility and to improve the rate recovery. The extraction efficiency is influenced by $\mathrm{pH}$. Bisoprolol and metoprolol have $\mathrm{pKa}$ values above 9, at low values of $\mathrm{pH}$ may occur the protonation of drugs, while at high values of $\mathrm{pH}$ may occur the hydrolysis of the selective beta-blockers [29].

\section{Protein precipitation technique}

Protein precipitation is frequently used to solubilise any bound drug and to precipitate any protein, is a technique characterized by simplicity and universality, but the interferences can be high [11]. This technique has been applied for the separation of metoprolol and simvastatin, the purification and pre-concentration of plasma samples being performed. The lower limit of quantification was about 1 $\mathrm{ng} / \mathrm{mL}$ for metoprolol. The extraction recovery was found between $87.32 \pm 3.21 \%$ and $90.11 \pm 3.64 \%$. The comparison between peak areas from plasma samples spiked before extraction and peak areas from plasma samples extracted and spiked after extraction allowed the determination of the extraction efficiency of metoprolol [7]. Acetonitrile was used as solvent for precipitation of proteins from human plasma samples containing bisoprolol fumarate and hydrochlorothiazide. For different levels of concentration the recovery rate for bisoprolol was between 90.70-101.7\% [25]. Bisoprolol was also separated from the proteins in human plasma by protein precipitation with acetonitrile, then analyzed by liquid chromatography coupled with mass spectrometry using diphenhydramine as internal standard. This technique applied for biological sample preparation provided a good recovery of the analyte, with values of $92.7 \pm 8.1,94.8 \pm 4.9$ and $95.9 \pm 3 \%$ at concentration of 1 , 10 and $80 \mathrm{ng} / \mathrm{mL}$ [12]. Protein precipitation technique was applied for the separation of bisoprolol in human plasma, with a recovery rate of about 94.03, 100.3 and $96.29 \%$ for $1.5,40$ and $80 \mathrm{ng} / \mathrm{mL}$ of bisoprolol, respectively. After analyzing the samples no interference normally present in plasma was detected[13].

\section{Liquid-liquid extraction}

Liquid-liquid extraction is a commonly used technique for the separation of metoprolol and bisoprolol in biological samples, by which the sample is purified and concentrated[11]. It involves an uneven distribution of solutes between two immiscible liquid phases. The most widely used solvents for the extraction of metoprolol and bisoprolol are methanol, dichloromethane, ether, acetone, benzene, toluene [33]. This technique is laborious, time-consuming and requires rather large quantities of toxic solvents. Usually, the resulting extract is evaporated to dryness and the residue isredissolved in a suitable solvent before the application of the analytical method [29]. Some important works describing the liquid-liquid extraction of metoprolol and bisoprolol in biological fluids are summarized below (Table I). 
TABLE I. Preparation of biological samples containing metoprolol and bisoprolol by liquid-liquid extraction technique

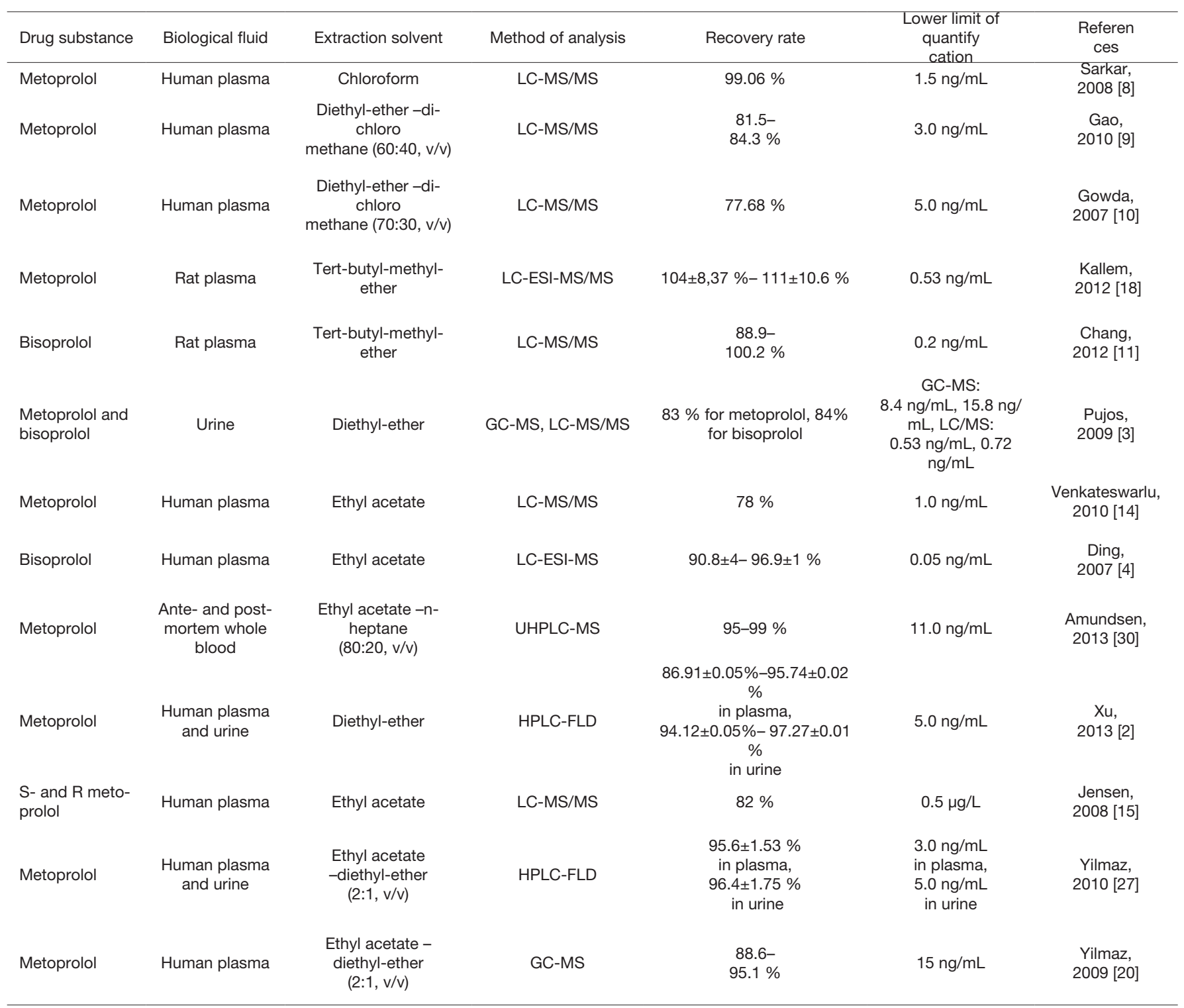

\section{Liquid-liquid microextraction}

Determination of metoprolol with other analytes in human plasma samples was performed using high performance liquid chromatography coupled with ultraviolet detection as the method for quantitative determination. Biological samples were processed using dispersive liquid-liquid microextraction and the separation of analytes was performed using isocratic elution with ultraviolet detection. The relative recovery of metoprolol was between 94-104\% [29]. For the analysis of four beta-blockers from human urine, bisoprolol included, a new method, namedcarrier-mediated liquid phase microextraction coupled with high performance liquid chromatography has been developed. The limit of detection for bisoprolol was about $0.01 \mathrm{mg} / \mathrm{mL}$, with a relative recovery of about $100.8 \%$ for $1.0 \mathrm{mg} / \mathrm{L}$ and $100.3 \%$ for $5 \mathrm{mg} / \mathrm{L}$ [24]. Metoprolol and other drugs were separated from biological fluids using hollow fibre-protected liquid phase microextraction, followed by analysis of drug substances by gas chromatography-mass spectrometry. The processing method lead to satisfactory results for the separation of metoprolol from human urine samples obtained following a single dose administration. The extraction recovery was found between 93.79-109.04\%[21].

\section{Solid phase extraction}

In solid phase extraction, the selected beta-blockers are distributed between a solid phase and a liquid phase, having a greater affinity for the solid phase [34]. It involves the use of extraction cartridges and is a fast and selective technique used for processing biological samples. This technique provides good separation of drug substances from interferences, high recovery rates for metoprolol and bisoprolol,and requires a reduced consumption of organic solvents, things that represent advantages when compared to the other techniques applied for sample preparation [29].

Solid phase extraction technique was applied for extraction of metoprolol in plasma samples obtained from pediatric patients. The samples were filtered through Bond-Elut columns under vacuum, then washed twice withdouble distilled water and eluted with methanol. The recovery rate 
of metoprolol was of about $73.0 \pm 20.5 \%$ [23]. In addition, 96-well pipette tips with a chemically bonded monolithic sorbent plug were used for the solid phase extraction of metoprolol and pindolol in human plasma samples. Ropivacaine was used as internal standard. Finally, the analytes were eluted into a clean 96-well plate with methanol in water. The low limit of quantification was between $94 \%$ and $108 \%$, and the accuracy was from $101 \%$ to $103 \%$ for metoprolol [16]. Bisoprolol was extracted from human plasma using solid phase extraction technique and metoprolol as internal standard. The human plasma sample containing the selected medicine and internal standard was processed using Oasis HLB extraction cartridge, then eluted with acetonitrile. The recovery of bisoprolol was $93.98 \%$ with an accuracy of $4.46 \%$ [17]. To extract (S)-(-) and (R)-(+)metoprolol the LiChrosep DVB HL extraction cartridge employing $200 \mu \mathrm{L}$ of human plasma was used. The sample was first washed with water, then with methanol: $2 \%$ ammonia solution in water $(30: 70)(\mathrm{v} / \mathrm{v})$. The analytes were eluted with acetic acid in methanol. The recovery rate for both the enantiomers was greater than 94\% [19]. Liquid chromatography coupled with mass spectrometry was used to simultaneously determine several beta-blockers, metoprolol and bisoprolol included, in post-mortem whole blood. Sample preparation was done using automated solid phase extraction. Oasis MCX extraction cartridge which is a mixed-mode, cation-exchange,reversed-phase sorbent was used [26]. Oasis MCX, WAX, MAX and WCX extraction cartridges have been used for the solid phase extraction of metoprolol and others beta-blockers from urine samples. Samples were washed with $2 \%$ formic acid or ammonium hydroxide in water $(5: 95, \mathrm{v} / \mathrm{v})$ depending on the analyte and cartridge used [6]. Separation of (S)-(-) and (R)-(+)bisoprolol in human plasma samples was performed using C18 cartridge. The recovery rate for the two enantiomers was in the range of $95-102 \%$, and the limit of detection was $20 \mathrm{ng} / \mathrm{mL}$ and $5 \mathrm{ng} / \mathrm{mL}$ respectively [28].

On-line molecularly imprinted solid phase extraction is a new technique that has been applied to urine samples that contained beta-blockers, metoprolol included. The column filled with the molecularly imprinted polymer was coupled to a multi-dimensional LC-MS/MS, and the mixture with $0.01 \%$ aqueous solution of formic acid-methanol $(30: 70, v / v)$ was used for elution [32].

\section{Conclusions}

The main objective of this work was to present the most frequently used techniques for the preparation of biological samples containing metoprolol and bisoprolol and also to present several analytical methods applied for determination of the selected beta-blockers. Liquid-liquid extraction, solid phase extraction technique, respectively the modern methods of separation such as dispersive liquidliquid microextraction, carrier-mediated liquid phase or hollow fibre-protected liquid phase microextraction shows high levels of recovery, indicating that these can be suc- cessfully applied for the separation of metoprolol and bisoprolol from biological samples. For a better separation it is important to choose an appropriate extraction solvent in a suitable volume and a proper $\mathrm{pH}$ considering the basic character of the two analytes. After the extraction process, the analysis of metoprolol and bisoprolol in human plasma, urine and other biological fluids through different chromatographic methods provides important information in clinical and toxicological studies.

\section{Acknowledgement}

This work was supported by the "Program of Excellence in doctoral and postdoctoral multidisciplinary research in chronic diseases", contract no. POSDRU/159/1.5/S/133377, financed from the European Social Fund through the Sectoral Operational Programme for Human Resources Development 2007-2013.

\section{References}

1. Katalin M. New therapeutic perspectives in hypertension treatment. Acta Medica Marisiensis. 2012; 58(2):124-130.

2. $\mathrm{Xu} \mathrm{T,} \mathrm{Bao} \mathrm{S,} \mathrm{Geng} P$ et al. Determination of metoprolol and its two metabolites in human plasma and urine by high performance liquid chromatography with fluorescence detection and its application in pharmacokinetics. J Chromatogr B. 2013;937:60-66.

3. Pujos E, Cren-Olive C, Paisse O, Flament-Waton M, Grenier-Loustalot MF. Comparison of the analysis of $\beta$-blockers by different techniques. J Chromatogr B. 2009;877:4007-4014.

4. Ding L, Zhou X, Guo X, Song Q, He J, Xu G. LC-ESI-MS method for the determination of bisoprolol in human plasma. J Pharm Biomed Anal. 2007;44:520-525

5. Li N, Li C, LU N, Dong Y. A novel micellar per aqueous liquid chromatographic method for simultaneous determination of diltiazem hydrochloride, metoprolol tartrate and isosorbide mononitrate in human serum. J Chromatogr B.2014;967:90-97.

6. Marchi I, Rudaz S, Veuthey J. Sample preparation development and matrix effects evaluation for multianalyte determination in urine. J Pharm Biomed Anal. 2009;49:459-467.

7. Selvan PS, Pal TK. Chromatography-tandem mass spectrometry method for the simultaneous quantitation of metoprolol succinate and simvastatin. J Pharm Biomed Anal. 2009;49:780-785.

8. Sarkar AK, Ghosh D, Das A, et al. Simultaneous determination of metoprolol succinate and amlodipine besylate in human plasma by liquid chromatography - tandem mass spectrometry and its application in bioequivalence study. J Chromatogr B. 2008;873:77-85.

9. Gao F, Zhang M, Cui X, Wang Z, Sun Y, Gu J. Simultaneous quantitation of hydrochlorothiazide and metoprolol in human plasma by liquid chromatography-tandem mass spectrometry. J Pharm Biomed Anal.2010;52:149-154.

10. Gowda KV, Mandal U, Selvan PS et al. Liquid chromatography tandem mass spectrometry method for simultaneous determination of metoprolol tartrate and ramipril in human plasma. J Chromatogr B. 2007;858:13-21.

11. Chang H, Li J, Li J et al. Simultaneous determination of amlodipine and bisoprolol in rat plasma by a liquid chromatography/tandem mass spectrometry method and its application in pharmacokinetic study. J Pharm Biomed Anal. 2012;71:104-110.

12. Liu M, Zhang D, Sun Y, Wang Y, Liu Z, Gu J. Liquid-chromatographicelectrospray tandem mass spectrometric determination of bisoprolol in human plasma. Biomed Chromatogr. 2007;21:508-513.

13. Liu G, Wang W, Jia J et al. Liquid chromatography tandem mass spectrometry method for determination of bisoprolol in human plasma using d5-bisoprolol as the internal standard. Biomed Chromatogr. 2010;24:574-580

14. Venkateswarlu P, Kumar B, Seshaiah K, Prasad W. Selective and sensitive method for the determination of metoprolol in human plasma using liquid chromatography coupled with tandem mass spectrometry. Acta Pharm. 2010;60:177-184.

15. Jensen BP, Sharp CF, Gardiner SJ, Begg EJ. Development and validation of a stereoselective liquid chromatography-tandem mass 
spectrometry assay for quantification of S- and R-metoprolol in human plasma. J Chromatogr B. 2008;865:48-54.

16. Abdel-Rehim M, Persson C, Altun Z, Blomberg L. Evaluation of monolithic packed 96-tips and liquid chromatography-tandem mass spectrometry for extraction and quantification of pindolol and metoprolol in human plasma samples. J Chromatogr A.2008;1196-1197:23-27.

17. Bhatt J, Subbaiah $G$, Kambli $S$ et al. A high throughput and sensitive liquid chromatography-tandem mass spectrometry (LC-MS/MS) method for the estimation of bisoprolol in human plasma using multiplexing technique. J Chromatogr B.2007; 852:374-381.

18. Kallem RR, Ramesh M, Seshagirirao JVLN. Validated LC-ESI-MS/MS method for simultaneous quantitation of felodipine and metoprolol in rat plasma: application to a pharmacokinetic study in rats. Biomed Chromatogr. 2013;27:784-791.

19. Sharma P, Contractor P, Guttikar S, Patel DP, Shrivastav PS Development of a sensitive and rapid method for quantification of (S)$(-)$ and (R)-(+)-metoprolol in human plasma by chiral LC-ESI-MS/MS. J Pharm Anal. 2014;4(1):63-79.

20. Yilmaz B, Arslan S, Akba V. Gas chromatography-mass spectrometry method for determination of metoprolol in the patients with hypertension. Talanta. 2009;80:346-351.

21. Liu W, Zhang L, Wei Z, Chen S, Chen G. Analysis of $\beta$-agonists and $\beta$-blockers in urine using hollow fibre-protected liquid-phase microextraction with in situ derivatizationfollowed bygas chromatography/ mass spectrometry. J Chromatogr A. 2009;1216:5340-5346.

22. Liu W, Yan Z, Huang $X$ et al. Simultaneous determination of blockers and agonists

23. by on-fiber derivatization in self-made solid-phase microextraction coating fiber. Talanta. 2015;132:915-921.

24. Albers S, Elshoff J, Völker C, Richter A, Läer S. HPLC quantification of metoprolol with solid-phase extraction for the drug monitoring of pediatric patients. Biomed Chromatogr. 2005;19:202-207.

25. Zhang L, Su X, Zhang C, et al. Extraction and preconcentration of ?-blockers in human urine for analysis with high performance liquid chromatography by means of carrier-mediated liquid phase microextraction. Talanta. 2010;82:984-992.

26. Tutunji MF, Ibrahim HM, Khabbas MH, Tutunji LF. Simultaneous determination of bisoprolol and hydrochlorothiazide in human plasma by HPLC coupled with mass spectrometry. J Chromatogr B. 2009;877:1689-1697.

27. Kristoffersen L, Øiestad EL, Opdal MS, Krogh M, Lundanes E, Christophersen AS. Simultaneous determination of 6 beta-blockers, 3 calcium-channel antagonists, 4 angiotensin- $\|$ antagonists and 1 antiarrhytmic drug in post-mortem whole blood by automated solid phase extraction and liquid chromatography mass spectrometry. J Chromatogr B. 2007;850:147-160.

28. Yilmaz B, Asci A, Arslan S. Determination of metoprolol in human plasma and urine by high-performance liquid chromatography with fluorescence detection. J Sep Sci. 2010;33:1904-1908.

29. Hefnawy MM, Sultan MA, Al-Shehri MM. Enantioanalysis of bisoprolol in human plasma with a macrocyclic antibiotic HPLC chiral column using fluorescence detection and solid phase extraction. Chem Pharm Bull. 2007;55(2):227-230

30. Jouyban A, Sorouraddin MH, Farajzadeh MA, Somi MH, FazeliBakhtiyari R. Determination of five antiarrhytmic drugs in human plasma by dispersive liquid-liquid microextraction and high-performance liquid chromatography. Talanta. 2015;134:681-689.

31. Amundsen I, Øiestad A, Ekeberg D, Kriestoffersen. Quantitative determination of fifteen basic pharmaceuticals in ante- and postmortem whole blood by high $\mathrm{pH}$ mobile phase reversedphase ultra high performance liquid chromatography-tandem mass spectrometry. J Chromatogr B. 2013;927:112-123.

32. Spietelun A, Marcinkowski L, Guardia M, Namieśnik J. Green aspects, developments and perspectives of liquid phase microextraction techniques. Talanta. 2014;119:34-45.

33. Santos MG, Tavares IM, Boralli VB, Figueiredo EC. Direct doping analysis of beta-blocker drugs from urinary samples by on-line molecularly imprinted solid-phase extraction coupled to liquid chromatography/ mass spectrometry. Analyst. 2015 Feb 13. [Epub ahead of print]

34. Saleem K, Ali I, Kulsum U, Aboul-Enein HY. Recent developments in HPLC analysis of B-blockers in biological samples. J Chromatogr Sci. 2013;51(8):807-818

35. Fontanals N, Marcé RM, Borrull F. New hydrophilic materials for solidphase extraction. Trends Anal Chem. 2005;24(5):394-406. 